\title{
ACTITUD DE LOS PADRES HACIA LA POSIBILIDAD DE EMPRENDIMIENTO DE SUS HIJOS. FACTORES VINCULADOS
}

\author{
Rosa Ana Alonso Ruiz \\ Ana Ponce de León Elizondo \\ Universidad de La Rioja \\ Juan Alfredo Jiménez Eguizabal \\ Universidad de Burgos
}

RESUMEN: El emprendimiento en la sociedad actual goza de un papel relevante como generador de empleo, así como motor de crecimiento económico y de innovación. Aportes, todos ellos, que destacan el papel esencial de la educación para la formación de personas emprendedoras. El artículo tuvo como objetivo vincular factores como el sexo, el nivel de estudios y la situación profesional de los padres que tienen hijos en la etapa postobligatoria, con la actitud que presentan ante la situación de que su descendiente se decidiera a crear su propia empresa y las ayudas que le proporcionaría. Se utilizaron pruebas paramétricas T de Student y varianza de un factor (ANOVA) con una muestra de 791 progenitores. Los resultados presentan que son los padres con estudios primarios y en activo, que trabajan tanto por cuenta propia como ajena, los que más desean que sus hijos e hijas monten una empresa. Las conclusiones de este estudio apuntan a fortalecer la educación y la formación en el emprendimiento, especialmente en el ámbito familiar, para crear, educar, construir y producir futuros emprendedores.

PALABRAS CLAVE: Emprendimiento, familia, jóvenes, educación secundaria.

\section{ATTITUDE OF PARENTS TOWARDS THE CHANCE OF ENTREPRENEURSHIP OF THEIR CHILDREN. RELATED FACTORS}

ABSTRACT: Entrepreneurship in today's society has a relevant role as a generator of employment, as well as an engine for economic growth and innovation. Contributions, all of them, which highlight the essential role of education for the training of entrepreneurs. The article aimed to link factors 
such as gender, level of education and professional situation of parents, who have children in the post-compulsory stage, with the attitude they present to the situation of their descendant deciding to create his own company, as well as the aid it would provide. T Student's parametric tests and one-factor (ANOVA) were used with a sample of 791 progenitors. The results show that they are male parents with primary education and that they work for their own account or others who most want their children to set up a company. The conclusions of this study aim to strengthen education and training in entrepreneurship, especially in the family, to create, educate, build and produce future entrepreneurs.

KEYWORDS: Entrepreneurship, family, youth, Secondary education.

Recibido: $31 / 07 / 2017$

Aceptado: 08/09/2017

Correspondencia: Rosa Ana Alonso Ruiz, Universidad de La Rioja, C/ Luis de Ulloa, s/n, 26004 Logroño. Email: rosa-ana.alonso@unirioja.es.

\section{INTRODUCCIÓN}

En la última década, la estimulación del espíritu emprendedor ha sufrido un importante auge, como aporte tanto en la generación de empleo como en el crecimiento económico y la innovación (Alemany, Alvarez, Planellas y Urbano, 2011). Se destaca, por un lado, a nivel estatal, que en 2013 se configuró la Ley 14/2013, de apoyo a los emprendedores y su internacionalización; asimismo a nivel autonómico, algunas Comunidades Autónomas han desarrollado normativas de ayuda al emprendimiento (Castilla La Mancha, 2011; Comunidad Valenciana, Islas Baleares y País Vasco, 2012; Murcia y Navarra, 2013).

Por otro lado, desde un punto de vista educativo, el progreso de la importancia del emprendimiento en la educación es notorio y ha sido reavivado como variable explicativa de la política laboral y de la problemática empresarial (Jiménez, Palmero y Jiménez, 2012; Flores y Palao, 2013; Mwasalwiba, 2010; Osorio y Pereira, 2011; Von Graevenitz, Harhoff y Weber, 2010), al considerar la educación un medio idóneo para lograr la transformación y el cambio de la sociedad (Orrego, 2009). De esta circunstancia se ha hecho eco la actual ley educativa, la Ley Orgánica de Mejora de la Calidad Educativa (2013) (en adelante LOMCE) al establecer que la educación resulta un elemento esencial en la formación de personas emprendedoras, así lo refleja uno de sus principales objetivos denominado desarrollar el espíritu emprendedor de los estudiantes, además estos resultados de aprendizaje deberán ser trabajados en todas las áreas; pero concretamente en la etapa de Educación Secundaria Obligatoria contempla el desarrollo de la asignatura Iniciación a la Actividad Emprendedora y Empresarial.

Parece claro que la literatura emprendedora ha puesto de manifiesto que la educación es una herramienta indispensable y elemento complementario del aprendizaje para que el alumnado disponga de un conocimiento más profundo sobre 
sus posibilidades de emprender (Flores y Palao, 2013; Von Graevenitz, Harhoff y Weber, 2010) y en la formación de competencias emprendedoras (Vican y Luketić, 2007); pero dentro de ese proceso educativo existen diversos agentes implicados. Algunas investigaciones revalorizan el papel de la educación destacando el rol del profesor en este quehacer (De la Torre, Ruiz, Escolar, Palmero y Jiménez, 2016; Seikkula-Leino, Ruskovaara, Ikavalko, Mattila y Rytkola, 2010), pero además en la edad juvenil es necesario tener presente otro agente fundamental, la familia como sistema formalizado, abierto, propositivo y autorregulado (Álvarez y Rodríguez, 2008) y, en la actualidad, el capital familiar es una forma de capital social para sus miembros, así como para el funcionamiento de la economía y de la sociedad (Bubolz, 2001).

Se afirma que el espíritu empresarial puede ser aprendido y desarrollado, por lo que la educación recibida en los primeros años puede ser esencial para crear las bases de habilidades, capacidades y valores necesarios para emprender (Carlos, Contreras, Silva y Liquidano, 2015; Vican y Luketić, 2007).

Las investigaciones que han reseñado el papel de la familia lo han desarrollado, en la mayoría de las ocasiones, para explicar la influencia en el comportamiento de los emprendedores (Cuadrado y García, 2006; Davidsson y Honig, 2003), aludiendo a valores personales, tales como la sobreconfianza, la percepción del riesgo o la creatividad, entre otros (Espíritu, 2011; Taktak y Triki, 2015); así como para indagar en el funcionamiento y mantenimiento de empresas familiares (Arregle, Hitt, Sirmon y Very, 2007), analizando factores como el apoyo familiar recibido, tanto a nivel económico como relacional, la existencia de antecedentes empresariales, etc. (Bubolz, 2001; Santamaría, Moreno, Torres y Cadrazco, 2013).

El presente artículo se centra en la familia, especialmente en aquellas que tienen hijos en la etapa postobligatoria, dado que este colectivo juvenil está en puertas del inicio de su actividad profesional. Se pretende vincular factores como el sexo, el nivel de estudios y situación profesional de los progenitores con la actitud de estos ante la situación de que su hijo se decidiera a crear su propia empresa, así como las ayudas que le proporcionaría.

\section{Metodología}

\section{Población y muestra}

La población estuvo constituida por las familias que tienen hijos cursando Educación Secundaria Postobligatoria del estado español censados durante el curso 2013-2014. El tamaño muestral definitivo ascendió a 791 progenitores. De los 791 padres, el $70.8 \%$ eran mujeres $(n=560)$ y el $29.2 \%$ hombres $(n=231)$. Casi el $40 \%$ de los ascendentes disponen de estudios secundarios (Bachiller o FP), mientras que el $34.3 \%$ posee estudios superiores universitarios, el $25.4 \%$ estudios primarios (EGB) y tan sólo un $1 \%$ de los progenitores no dispone de estudios.

En cuanto a la situación profesional, 6 de cada 10 familiares está empleado por cuenta ajena (58.4\%), frente a un $14.2 \%$ que lo está por cuenta propia, un $12 \%$ se encuentra en situación de desempleo, un $11.6 \%$ se dedica a la atención del hogar, un $2.8 \%$ es pensionista y un $1 \%$ jubilado. 
Se realizó un muestreo aleatorio simple de la población, por afijación proporcional en cada una de las 6 áreas en las que se dividió al territorio español, más Ceuta y Melilla. Las 6 áreas fueron las siguientes:

\begin{tabular}{|l|l|}
\hline Denominación/Localización & Comunidades y/o provincias \\
\hline A1-Noreste & Cataluña, Aragón (excepto Teruel) y Baleares \\
\hline A2-Levante & Comunidad Valenciana, Murcia y Albacete \\
\hline A3-Sur & Andalucía, Islas Canarias, Ceuta y Melilla \\
\hline A4-Sur & $\begin{array}{l}\text { Madrid, Castilla-La Mancha (excepto Albacete), Castilla-León } \\
\text { (excepto León, Palencia y Burgos), Cáceres y Teruel }\end{array}$ \\
\hline A5-Noroeste & Galicia, Asturias y León \\
\hline A6-Norte & Cantabria, País Vasco, La Rioja, Navarra, Burgos y Palencia \\
\hline
\end{tabular}

Figura 1. Agrupación territorial para la muestra. Fuente: Elaboración propia

\section{Variables}

Se establecen 3 variables que puntualizan las características generales del progenitor y 2 variables instrumentales que definen la deseabilidad de emprendimiento de estos familiares hacia sus hijos. A continuación, se detallan cada una de ellas:

- Género del progenitor: variable dicotómica que identifica si el encuestado es hombre o mujer.

- Nivel de estudios: variable que recoge el nivel de estudios: superiores universitarios, secundarios, primarios y sin estudios.

- Situación profesional: variable que atiende a categorías como: empleado por cuenta ajena, trabajador a cuenta propia, atención del hogar, parado, pensionista o jubilado.

- Grado en que le gustaría que su hijo se decidiera a crear su propia empresa: variable que pretende valorar dicha cuestión.

- Grado en que ayudaría a su hijo si se decidiera a crear su propia empresa: variable que pretende puntuar este aspecto y, además, valorar la ayuda que les proporcionaría:

- Apoyo económico

- Apoyo moral

- Aportando ideas

- Otro tipo de ayuda, ¿cuál?

\section{Instrumento}

Los ítems de los que se sirve este estudio formaban parte de un cuestionario más amplio integrado por diversas secciones temáticas y recogido dentro del proyecto "RESORTES" del Plan Nacional I+D+i con referencia EDU2012-39080-C07-00. La validación se realizó mediante una prueba piloto de la que formaron parte 8 comunidades autónomas, así como un análisis de expertos en el que participaron 14 investigadores de siete universidades españolas. 
Se analiza la información recogida con los ítems 5, 17 y 18. La pregunta 5 registraba el nivel de estudios y la situación profesional del progenitor, el ítem 17 recogía el grado en que le gustaría que su hijo se decidiera a crear su propia empresa y el ítem 18 preguntaba sobre el grado en que el progenitor ayudaría a su hijo si se decidiera a crear su propia empresa, además se debía valorar si esa ayuda respondería a un apoyo económico, a un apoyo moral, aportando ideas u otro tipo de ayuda. A estos dos últimos ítems se debía responder a través de una escala tipo Likert de 5 puntos con los siguientes valores: 1 "nada", 2 "poco", 3 "algo", 4 "bastante" y 5 "mucho".

\section{Procedimiento}

La aplicación del cuestionario se realizó de forma aleatoria entre los progenitores de los distintos centros educativos de cada uno de los seis territorios en los que se ha dividido el estado español. Se solicitó permiso y se les informó de los pormenores de la investigación al Director General de Educación de cada Comunidad Autónoma, así como a los directores de los centros educativos, antes de la aplicación del instrumento. Para la administración del cuestionario, dos investigadores debidamente formados acudieron personalmente a cada centro para informar directamente a los padres sobre la cumplimentación del cuestionario, intentando reducir, de este modo, la mortalidad experimental.

\section{Análisis de resultados}

Se ha conformado el análisis de los datos en dos fases. En la primera, se generó un análisis descriptivo sobre características personales de los progenitores referentes al sexo, al nivel de estudios y a la situación profesional, así como sobre el grado en que le gustaría que su hijo decidiera montar su propia empresa y en qué le ayudaría (apoyo económico, moral, aportando ideas o de otro tipo) y se calculó la media y su desviación estándar para cada una de las variables.

En la segunda fase se llevó a cabo un análisis inferencial a través de dos pruebas:

- La prueba paramétrica T de Student para muestras independientes, con el propósito de determinar si existían diferencias en el grado en que les gustaría que su hijo se decidiera a montar su propia empresa y en el grado en que le ayudaría en relación con el sexo del progenitor.

- La prueba de varianza de un factor (ANOVA de un factor) permitió acreditar la existencia de diferencias en el grado en que les gustaría que su hijo se decidiera a montar su propia empresa y en el grado en que le ayudaría en función del nivel de estudios o la situación profesional de los progenitores. En todo análisis de varianza se probó la homogeneidad de las varianzas con el fin de acreditar los supuestos de normalidad y homocedasticidad. Finalmente, se efectuaron contrastes mediante comparaciones múltiples post-hoc; cuando el estadístico de Levene asumió varianzas iguales se empleó la prueba HSD Tukey, mientras que en los casos que el estadístico de Levene no asumió varianzas iguales, se utilizó la prueba Games-Howell. El nivel de significación considerado en todo momento fue $\mathrm{p}<0.05$. 


\section{Resultados}

Son los padres $(\bar{X}=3.79 \pm 1.101)$ los que más les gustaría que sus hijos montaran una empresa frente a las madres $(\bar{X}=3.51 \pm 1.135)$, asimismo le ayudarían, en mayor medida, de forma económica (padres $(\bar{X}=3.76 \pm 1.125)$; madres $(\bar{X}=3.56 \pm 1.178)$ ). Sin embargo, no existen diferencias significativas entre progenitores masculinos y femeninos y otros factores como el grado en que ayudaría a su hijo a montar su propia empresa mediante apoyo moral, aportando ideas u otro tipo de ayudas (Tabla 1).

Tabla 1. Resumen Prueba T para muestras independientes: Grado en que a los progenitores les gustaría que su hijo se decidiera a montar su propia empresa y en qué le ayudaría (apoyo económico, moral, aportando ideas u otro tipo de ayuda)

\begin{tabular}{|c|c|c|c|c|c|c|}
\hline \multicolumn{2}{|l|}{ Grado en que... } & \multirow{3}{*}{$\begin{array}{c}\begin{array}{r}\text { Estadístico } \\
\text { de Levene }\end{array} \\
1.561\end{array}$} & \multirow{3}{*}{$\begin{array}{c}\boldsymbol{p} \\
.212\end{array}$} & \multirow{2}{*}{$\begin{array}{c}\boldsymbol{t} \\
-3.092\end{array}$} & \multirow{2}{*}{$\frac{\mathbf{g l}}{723}$} & \multirow{2}{*}{$\frac{\boldsymbol{p}}{.002^{*}}$} \\
\hline Le gustaría que su hijo/a se & $\begin{array}{l}\text { Se asumen } \\
\text { varianzas iguales }\end{array}$ & & & & & \\
\hline empresa & $\begin{array}{l}\text { No se asumen } \\
\text { varianzas iguales }\end{array}$ & & & -3.130 & 413 & \\
\hline \multirow{2}{*}{$\begin{array}{l}\text { Ayudaría a su hijo/a a montar su } \\
\text { propia empresa }\end{array}$} & $\begin{array}{l}\text { Se asumen } \\
\text { varianzas iguales }\end{array}$ & 3.018 & .083 & -1.310 & 633 & .191 \\
\hline & $\begin{array}{l}\text { No se asumen } \\
\text { varianzas iguales }\end{array}$ & & & -1.392 & 416 & \\
\hline \multirow{2}{*}{$\begin{array}{l}\text { Ayudaría a su hijo/a } \\
\text { APOYO ECONÓMICO }\end{array}$} & $\begin{array}{l}\text { Se asumen } \\
\text { varianzas iguales }\end{array}$ & .814 & .367 & -2.166 & 758 & $.031 *$ \\
\hline & $\begin{array}{l}\text { No se asumen } \\
\text { varianzas iguales }\end{array}$ & & & -2.206 & 448 & \\
\hline \multirow{2}{*}{$\begin{array}{l}\text { Ayudaría a su hijo/a } \\
\text { APOYO MORAL }\end{array}$} & $\begin{array}{l}\text { Se asumen } \\
\text { varianzas iguales }\end{array}$ & .033 & .856 & -.030 & 769 & .976 \\
\hline & $\begin{array}{l}\text { No se asumen } \\
\text { varianzas iguales }\end{array}$ & & & -.030 & 445 & \\
\hline \multirow{2}{*}{$\begin{array}{l}\text { Ayudaría a su hijo/a } \\
\text { APORTANDO IDEAS }\end{array}$} & $\begin{array}{l}\text { Se asumen } \\
\text { varianzas iguales }\end{array}$ & 2.725 & .099 & 1.171 & 767 & .242 \\
\hline & $\begin{array}{l}\text { No se asumen } \\
\text { varianzas iguales }\end{array}$ & & & 1.127 & 393 & \\
\hline \multirow{2}{*}{$\begin{array}{l}\text { Ayudaría a su hijo/a } \\
\text { OTRO TIPO DE APOYO }\end{array}$} & $\begin{array}{l}\text { Se asumen } \\
\text { varianzas iguales }\end{array}$ & .561 & .459 & -.443 & 32 & .661 \\
\hline & $\begin{array}{l}\text { No se asumen } \\
\text { varianzas iguales }\end{array}$ & & & -.519 & 25 & \\
\hline
\end{tabular}

Nota. $p<.05 ;{ }^{*}=p<.05$.

Fuente: Elaboración propia.

Los resultados del ANOVA de un factor, para el grado en que a los progenitores con hijos que cursen secundaria postobligatoria les gustaría que su hijo montara una empresa, así como el apoyo que les proporcionarían y, el nivel de estudios que posee el padre/madre, asumiendo en la mitad de los casos igualdad de las varianzas poblacionales analizadas, por lo que se utilizó el estadístico Games-Howell y en la otra mitad de las situaciones se asumen la igualdad de las varianzas por lo que se aplicó Tukey, se muestran diferencias significativas (Tabla 2). 
Tabla 2. Resumen ANOVA de un factor: Grado en que a los progenitores les gustaría que su hijo montara su propia empresa, así como en el grado que le ayudarían y, el nivel de estudios del padre/madre

\begin{tabular}{|c|c|c|c|c|c|c|c|c|}
\hline & \multirow{3}{*}{$\begin{array}{l}\text { Estadístico } \\
\text { de Levene }\end{array}$} & \multirow{3}{*}{$p$} & \multirow{2}{*}{\multicolumn{6}{|c|}{ ANOVA }} \\
\hline & & & & & & & & \\
\hline & & & & $\begin{array}{c}\text { Suma de } \\
\text { cuadrados }\end{array}$ & gl & $\begin{array}{c}\text { Media } \\
\text { cuadrática }\end{array}$ & $\mathrm{F}$ & $p$ \\
\hline \multirow{3}{*}{$\begin{array}{l}\text { Grado en que } \\
\text { le gustaría } \\
\text { que su hijo/a } \\
\text { se decidiera a } \\
\text { montar su propia } \\
\text { empresa }\end{array}$} & \multirow[b]{3}{*}{1,571} & \multirow[b]{3}{*}{, 195 } & Entre grupos & 15,528 & 3 & 5,176 & 4,104 &, $007^{*}$ \\
\hline & & & Dentro de grupos & 948,423 & 752 & 1,261 & & \\
\hline & & & Total & 963,951 & 755 & & & \\
\hline \multirow{3}{*}{$\begin{array}{l}\text { Grado en que } \\
\text { ayudaría a su } \\
\text { hijo/a a montar su } \\
\text { propia empresa }\end{array}$} & \multirow{3}{*}{1,963} & \multirow{3}{*}{,118 } & Entre grupos & 1,203 & 3 &, 401 & ,488 &, 691 \\
\hline & & & Dentro de grupos & 540,587 & 658 & 822 & & \\
\hline & & & Total & 541,790 & 661 & & & \\
\hline \multirow{3}{*}{$\begin{array}{l}\text { Grado en que } \\
\text { ayudaría a su } \\
\text { hijo/a APOYO } \\
\text { ECONÓMICO }\end{array}$} & \multirow{3}{*}{2,935} & \multirow{3}{*}{,033 } & Entre grupos & 15,769 & 3 & 5,256 & 3,907 &, $009 *$ \\
\hline & & & Dentro de grupos & 1061,477 & 789 & 1,345 & & \\
\hline & & & Total & 1077,246 & 792 & & & \\
\hline \multirow{3}{*}{$\begin{array}{l}\text { Grado en que } \\
\text { ayudaría a su } \\
\text { hijo/a APOYO } \\
\text { MORAL }\end{array}$} & \multirow{3}{*}{1,643} & \multirow{3}{*}{, 178} & Entre grupos & 557 & 3 & 186 &, 575 & 631 \\
\hline & & & Dentro de grupos & 258,407 & 801 &, 323 & & \\
\hline & & & Total & 258,964 & 804 & & & \\
\hline \multirow{3}{*}{$\begin{array}{l}\text { Grado en } \\
\text { que ayudaría } \\
\text { a su hijo/a } \\
\text { APORTANDO } \\
\text { IDEAS }\end{array}$} & \multirow{3}{*}{3,019} & \multirow{3}{*}{,029 } & Entre grupos & 4,781 & 3 & 1,594 & 2,014 &, 110 \\
\hline & & & Dentro de grupos & 631,375 & 798 & ,791 & & \\
\hline & & & Total & 636,156 & 801 & & & \\
\hline \multirow{3}{*}{$\begin{array}{l}\text { Grado en que } \\
\text { ayudaría a su } \\
\text { hijo/a OTRO } \\
\text { TIPO DE APOYO }\end{array}$} & \multirow{3}{*}{$6,238^{a}$} & \multirow{3}{*}{,005 } & Entre grupos & 9,217 & 3 & 3,072 & 2,891 & 051 \\
\hline & & & Dentro de grupos & 34,005 & 32 & 1,063 & & \\
\hline & & & Total & 43,222 & 35 & & & \\
\hline
\end{tabular}

a. Los grupos con sólo un caso se ignoran en el cálculo de la prueba de la homogeneidad de varianza para Grado en que ayudaría a su hijo/a OTRO TIPO DE APOYO.

Nota. $p<.05 ; *=p<.05$.

Fuente: Elaboración propia.

Al profundizar en estas diferencias significativas, a partir de comparaciones múltiples, en este caso, mediante la prueba de Tuckey, se pone de manifiesto que únicamente existen diferencias significativas entre aquellos progenitores que tienen estudios primarios y aquellos con estudios superiores ( $p=.049)$; de manera que aquellos que tienen estudios primarios $(\bar{X}=3.77 \pm 1.162)$ desean más que sus hijos se decidan a montar una empresa que aquellos que tienen estudios superiores ( $\bar{X}=3.49 \pm 1.055)$ (Tabla 3 ).

Si bien en principio existían diferencias significativas entre el apoyo económico que los progenitores proporcionarían a sus hijos para montar su propia empresa y el nivel de estudios de los padres/madres, tras los resultados obtenidos en la prueba de Games Howell no podemos determinar que haya diferencias significativas comparando dos a dos (Tabla 4). 
Tabla 3. Comparaciones múltiples mediante Tukey: Análisis del grado en que a los progenitores les gustaría que su hijo montara su propia empresa, mediante apoyo económico, y el nivel de estudios del padre/madre

\begin{tabular}{|c|c|c|c|c|c|c|}
\hline & & $\begin{array}{l}\text { (I) Nivel de } \\
\text { estudios }\end{array}$ & $\begin{array}{l}\text { (J) Nivel de } \\
\text { estudios }\end{array}$ & $\begin{array}{c}\text { Diferencia de } \\
\text { medias (I-J) }\end{array}$ & $\begin{array}{c}\text { Error } \\
\text { estándar }\end{array}$ & $P$ \\
\hline \multirow{12}{*}{$\begin{array}{l}\text { Grado en que } \\
\text { le gustaría } \\
\text { que su hijo/a } \\
\text { se decidiera a } \\
\text { montar su propia } \\
\text { empresa }\end{array}$} & \multirow{12}{*}{$\begin{array}{l}\text { HSD } \\
\text { Tukey }\end{array}$} & \multirow{3}{*}{ Ninguno } & Primarios (EGB) & ,804 &, 432 & 246 \\
\hline & & & $\begin{array}{l}\text { Secundarios } \\
\text { (Bachiller, FP) }\end{array}$ & 1,013 & ,429 & ,086 \\
\hline & & & $\begin{array}{l}\text { Superiores } \\
\text { Universitarios }\end{array}$ & 1,081 &, 430 & ,059 \\
\hline & & \multirow{3}{*}{ Primarios (EGB) } & Ninguno &,- 804 & 432 & 246 \\
\hline & & & $\begin{array}{l}\text { Secundarios } \\
\text { (Bachiller, FP) }\end{array}$ & ,209 & , 104 & , 189 \\
\hline & & & $\begin{array}{l}\text { Superiores } \\
\text { Universitarios }\end{array}$ &, $277^{*}$ & , 107 & ,049* \\
\hline & & \multirow{3}{*}{$\begin{array}{l}\text { Secundarios } \\
\text { (Bachiller, FP) }\end{array}$} & Ninguno & $-1,013$ & 429 & ,086 \\
\hline & & & Primarios (EGB) &,- 209 & , 104 & , 189 \\
\hline & & & $\begin{array}{l}\text { Superiores } \\
\text { Universitarios }\end{array}$ & ,068 & ,095 & ,891 \\
\hline & & \multirow{3}{*}{$\begin{array}{l}\text { Superiores } \\
\text { Universitarios }\end{array}$} & Ninguno & $-1,081$ & 430 & ,059 \\
\hline & & & Primarios (EGB) &,$- 277^{*}$ & , 107 & ,049 \\
\hline & & & $\begin{array}{l}\text { Secundarios } \\
\text { (Bachiller, FP) }\end{array}$ &,- 068 & ,095 & ,891 \\
\hline
\end{tabular}

Nota. $p<.05 ;^{*}=p<.05$. Fuente: Elaboración propia.

Tabla 4. Comparaciones múltiples mediante Games-Howell: Análisis del grado en que los progenitores ayudarían a su hijo a montar su propia empresa, mediante apoyo económico, y el nivel de estudios del padre/madre

\begin{tabular}{|c|c|c|c|c|c|c|}
\hline & & $\begin{array}{l}\text { (I) Nivel de } \\
\text { estudios }\end{array}$ & $\begin{array}{l}\text { (J) Nivel de } \\
\text { estudios }\end{array}$ & $\begin{array}{c}\text { Diferencia de } \\
\text { medias }(\mathrm{I}-\mathrm{J})\end{array}$ & $\begin{array}{l}\text { Error } \\
\text { estándar }\end{array}$ & $p$ \\
\hline \multirow{12}{*}{$\begin{array}{l}\text { Grado en que } \\
\text { ayudaría a su } \\
\text { hijo/a APOYO } \\
\text { ECONÓMICO }\end{array}$} & \multirow{12}{*}{$\begin{array}{l}\text { Games- } \\
\text { Howell }\end{array}$} & \multirow{3}{*}{ Ninguno } & Primarios (EGB) & $-1,082$ &, 438 & 156 \\
\hline & & & $\begin{array}{l}\text { Secundarios } \\
\text { (Bachiller, FP) }\end{array}$ & $-1,158$ & ,433 &, 124 \\
\hline & & & $\begin{array}{l}\text { Superiores } \\
\text { Universitarios }\end{array}$ & $-1,300$ &, 434 & ,083 \\
\hline & & \multirow{3}{*}{ Primarios (EGB) } & Ninguno & 1,082 &, 438 & , 156 \\
\hline & & & $\begin{array}{l}\text { Secundarios } \\
\text { (Bachiller, FP) }\end{array}$ &,- 076 & ,111 & ,900 \\
\hline & & & $\begin{array}{l}\text { Superiores } \\
\text { Universitarios }\end{array}$ &,- 218 &, 111 & 204 \\
\hline & & \multirow{3}{*}{$\begin{array}{l}\text { Secundarios } \\
\text { (Bachiller, FP) }\end{array}$} & Ninguno & 1,158 &, 433 & 124 \\
\hline & & & Primarios (EGB) &, 076 & ,111 & ,900 \\
\hline & & & $\begin{array}{l}\text { Superiores } \\
\text { Universitarios }\end{array}$ &,- 142 & ,093 & ,421 \\
\hline & & \multirow{3}{*}{$\begin{array}{l}\text { Superiores } \\
\text { Universitarios }\end{array}$} & Ninguno & 1,300 & 434 & ,083 \\
\hline & & & Primarios (EGB) & ,218 &, 111 & 204 \\
\hline & & & $\begin{array}{l}\text { Secundarios } \\
\text { (Bachiller, FP) }\end{array}$ & , 142 & ,093 & ,421 \\
\hline
\end{tabular}

Nota. $p<.05 ;^{*}=p<.05$. Fuente: Elaboración propia. 
Por su parte, los resultados del ANOVA de un factor, en cuanto al grado en que a los progenitores les gustaría que su hijo montara una empresa y en qué le ayudaría y, la situación profesional del padre/madre, asumen la igualdad de las varianzas poblacionales analizadas, por lo que se utilizó el estadístico Games-Howell, muestran diferencias significativas (Tabla 5).

Tabla 5. Resumen ANOVA de un factor: Grado en que a los progenitores les gustaría que su hijo montara su propia empresa y en qué le ayudaría y, la situación profesional del padre/madre

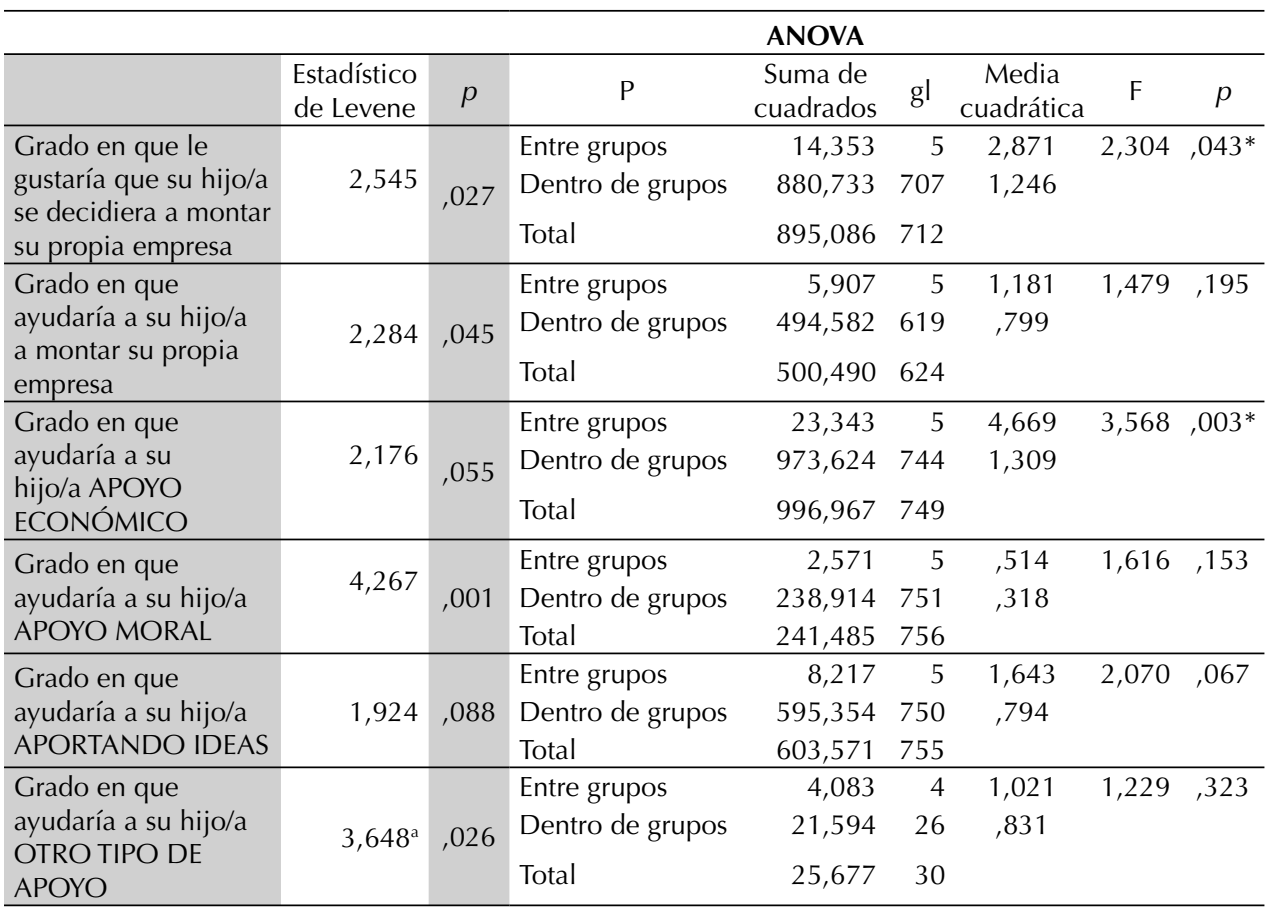

a. Los grupos con sólo un caso se ignoran en el cálculo de la prueba de la homogeneidad de varianza para Grado en que ayudaría a su hijo/a OTRO TIPO DE APOYO.

Nota. $p<.05 ;{ }^{*}=p<.05$. Fuente: Elaboración propia.

Tras el análisis post hoc de la prueba de Games-Howell se pone de manifiesto únicamente diferencias significativas entre el deseo de que sus hijos crearan su propia empresa de aquellos progenitores empleados por cuenta ajena y de aquellos que trabajan por cuenta propia $(p=.032)$. Los empleados por su propia cuenta $(\bar{X}=3.86$ \pm 1.010 ) desean más que sus hijos se decidan a montar una empresa que aquellos empleados por cuenta ajena ( $\bar{X}=3.51 \pm 1.093$ ) (Tabla 6).

Asimismo, existen diferencias significativas entre el apoyo económico que los padres proporcionarían a sus hijos y la situación profesional del padre/madre. Tras el análisis post hoc de la prueba de Tuckey se evidencian diferencias significativas entre aquellos progenitores empleados por cuenta ajena y los desempleados $(p=.014)$, siendo los empleados por cuenta ajena $(\bar{X}=3.64 \pm 1.102)$ los que aportarían a sus 
Tabla 6. Comparaciones múltiples mediante Games-Howell: Análisis del grado en que a los progenitores les gustaría que su hijo montara su propia empresa y la situación profesional del padre/madre

\begin{tabular}{|c|c|c|c|c|c|c|}
\hline & & $\begin{array}{l}\text { (I) Situación } \\
\text { profesional }\end{array}$ & $\begin{array}{l}\text { (J) Situación } \\
\text { profesional }\end{array}$ & $\begin{array}{c}\text { Diferencia de } \\
\text { medias (I-J) }\end{array}$ & $\begin{array}{c}\text { Error } \\
\text { estándar }\end{array}$ & Sig. \\
\hline & & & $\begin{array}{l}\text { Empleado/a por } \\
\text { cuenta propia }\end{array}$ &,$- 347^{*}$ & 114 &, $032 *$ \\
\hline & & Empleado/a por & Atención del hogar &,- 079 & , 131 & 991 \\
\hline & & cuenta ajena & Desempleado/a & ,020 & , 148 & 1,000 \\
\hline & & & Pensionista &,- 133 & ,301 & ,998 \\
\hline & & & Jubilado/a & 639 &, 518 & ,809 \\
\hline & & & $\begin{array}{l}\text { Empleado/a por } \\
\text { cuenta ajena }\end{array}$ &, $347^{*}$ & 114 & ,032 \\
\hline & & Empleado/a por & Atención del hogar &, 268 & , 156 &, 523 \\
\hline & & cuenta propia & Desempleado/a &, 367 & , 170 & ,265 \\
\hline & & & Pensionista & ,214 & ,313 & ,982 \\
\hline & & & Jubilado/a & ,986 &, 525 &, 477 \\
\hline & & & $\begin{array}{l}\text { Empleado/a por } \\
\text { cuenta ajena }\end{array}$ & ,079 & 131 & ,991 \\
\hline & & Atención del & $\begin{array}{l}\text { Empleado/a por } \\
\text { cuenta propia }\end{array}$ &,- 268 & , 156 &, 523 \\
\hline & & hogar & Desempleado/a & ,099 & 182 & ,994 \\
\hline en que le & & & Pensionista &,- 054 &, 320 & 1,000 \\
\hline gustaría que & & & Jubilado/a & ,718 &, 529 & ,750 \\
\hline $\begin{array}{l}\text { su hijo/a se } \\
\text { decidiera }\end{array}$ & $\begin{array}{l}\text { Games- } \\
\text { Howell }\end{array}$ & & $\begin{array}{l}\text { Empleado/a por } \\
\text { cuenta ajena }\end{array}$ &,- 020 & 148 & 1,000 \\
\hline $\begin{array}{l}\text { a montar } \\
\text { su propia }\end{array}$ & & Desempleado/a & $\begin{array}{l}\text { Empleado/a por } \\
\text { cuenta propia }\end{array}$ &,- 367 & , 170 & ,265 \\
\hline & & & Atención del hogar &,- 099 & , 182 & ,994 \\
\hline & & & Pensionista &,- 153 & ,327 & ,997 \\
\hline & & & Jubilado/a & ,619 &, 533 & ,843 \\
\hline & & & $\begin{array}{l}\text { Empleado/a por } \\
\text { cuenta ajena }\end{array}$ & ,133 & ,301 & ,998 \\
\hline & & Pensionista & $\begin{array}{l}\text { Empleado/a por } \\
\text { cuenta propia }\end{array}$ &,- 214 & ,313 & ,982 \\
\hline & & & Atención del hogar & ,054 & ,320 & 1,000 \\
\hline & & & Desempleado/a & , 153 & ,327 & ,997 \\
\hline & & & Jubilado/a &, 772 &, 594 & ,781 \\
\hline & & & $\begin{array}{l}\text { Empleado/a por } \\
\text { cuenta ajena }\end{array}$ &,- 639 &, 518 & ,809 \\
\hline & & Jubilado/a & $\begin{array}{l}\text { Empleado/a por } \\
\text { cuenta propia }\end{array}$ &,- 986 & ,525 & 477 \\
\hline & & & Atención del hogar &,- 718 &, 529 & ,750 \\
\hline & & & Desempleado/a &,- 619 & ,533 & ,843 \\
\hline & & & Pensionista &,- 772 &, 594 & ,781 \\
\hline
\end{tabular}

Nota. $p<.05 ; *=p<.05$.

Fuente: Elaboración propia. 
hijos mayor apoyo económico frente a los desempleados $(\bar{X}=3.19 \pm 1.401)$. También existen diferencias significativas entre aquellos progenitores empleados por cuenta propia y los desempleados $(p=.002)$, siendo los empleados por cuenta propia $(\bar{X}=$ $3.83 \pm 1.118$ ) los que aportarían mayor apoyo económico (Tabla 7).

Tabla 7. Comparaciones múltiples mediante Tukey: Análisis del grado en que a los progenitores ayudarían a su hijo a montar su propia empresa y la situación profesional del padre/madre

\begin{tabular}{|c|c|c|c|c|c|c|}
\hline & & $\begin{array}{l}\text { (I) Situación } \\
\text { profesional }\end{array}$ & (J) Situación profesional & $\begin{array}{l}\text { Diferencia } \\
\text { de medias } \\
(\mathrm{I}-\mathrm{J})\end{array}$ & $\begin{array}{c}\text { Error } \\
\text { estándar }\end{array}$ & $p$ \\
\hline \multirow{30}{*}{$\begin{array}{l}\text { Grado en que } \\
\text { ayudaría a su } \\
\text { hijo/a APOYO } \\
\text { ECONÓMICO }\end{array}$} & \multirow{30}{*}{$\begin{array}{l}\text { HSD } \\
\text { Tukey }\end{array}$} & \multirow{5}{*}{$\begin{array}{l}\text { Empleado/a por } \\
\text { cuenta ajena }\end{array}$} & $\begin{array}{l}\text { Empleado/a por cuenta } \\
\text { propia }\end{array}$ &,- 198 &, 122 &, 585 \\
\hline & & & Atención del hogar &, 067 & 135 & ,996 \\
\hline & & & Desempleado/a & $446^{*}$ & ,136 &, $014^{*}$ \\
\hline & & & Pensionista &,- 163 & ,262 & ,989 \\
\hline & & & Jubilado/a &, 512 & ,408 & 810 \\
\hline & & \multirow{5}{*}{$\begin{array}{l}\text { Empleado/a por } \\
\text { cuenta propia }\end{array}$} & Empleado/a por cuenta ajena & ,198 & , 122 & ,585 \\
\hline & & & Atención del hogar & ,265 & 165 &, 594 \\
\hline & & & Desempleado/a & ,644* & , 166 &, $002 *$ \\
\hline & & & Pensionista & 035 & ,278 & 1,000 \\
\hline & & & Jubilado/a & ,710 & 419 &, 536 \\
\hline & & \multirow{5}{*}{$\begin{array}{l}\text { Atención del } \\
\text { hogar }\end{array}$} & Empleado/a por cuenta ajena &,- 067 &, 135 & ,996 \\
\hline & & & $\begin{array}{l}\text { Empleado/a por cuenta } \\
\text { propia }\end{array}$ &,- 265 & , 165 &, 594 \\
\hline & & & Desempleado/a &, 379 &, 175 & ,257 \\
\hline & & & Pensionista &,- 230 & ,284 & ,966 \\
\hline & & & Jubilado/a &, 445 &, 423 & ,900 \\
\hline & & \multirow{5}{*}{ Desempleado/a } & Empleado/a por cuenta ajena &,$- 446^{*}$ & 136 & 014 \\
\hline & & & $\begin{array}{l}\text { Empleado/a por cuenta } \\
\text { propia }\end{array}$ &,$- 644^{*}$ &, 166 & ,002 \\
\hline & & & Atención del hogar &,- 379 &, 175 & ,257 \\
\hline & & & Pensionista &,- 610 & ,285 & ,267 \\
\hline & & & Jubilado/a &, 065 & 423 & 1,000 \\
\hline & & \multirow{5}{*}{ Pensionista } & Empleado/a por cuenta ajena &, 163 & ,262 & ,989 \\
\hline & & & $\begin{array}{l}\text { Empleado/a por cuenta } \\
\text { propia }\end{array}$ &,- 035 & ,278 & 1,000 \\
\hline & & & Atención del hogar &, 230 & ,284 & ,966 \\
\hline & & & Desempleado/a & 610 & ,285 & ,267 \\
\hline & & & Jubilado/a & 675 & ,479 & ,721 \\
\hline & & \multirow{5}{*}{ Jubilado/a } & Empleado/a por cuenta ajena &,- 512 & 408 & 810 \\
\hline & & & $\begin{array}{l}\text { Empleado/a por cuenta } \\
\text { propia }\end{array}$ &,- 710 & 419 &, 536 \\
\hline & & & Atención del hogar &,- 445 & ,423 &, 900 \\
\hline & & & Desempleado/a &,- 065 & ,423 & 1,000 \\
\hline & & & Pensionista &,- 675 & ,479 & ,721 \\
\hline
\end{tabular}

Nota. $p<.05 ; *=p<.05$.

Fuente: Elaboración propia. 


\section{Discusión}

Los resultados evidencian que son los progenitores masculinos con estudios primarios, bien que trabajan por cuenta propia como ajena, los que más desean que sus hijos monten una empresa, atendiendo a la afirmación de que en la familia se puede desarrollar el espíritu empresarial, lo que puede contribuir a mejorar su conocimiento sobre sus posibilidades de emprender (Von Graevenitz et al. 2010), así como asentar las raíces necesarias (habilidades, capacidades y valores) para este fin (Carlos et al., 2015). Cuestiones que se deberían contrastar y serían de gran interés para la comunidad científica con la percepción que tienen los hijos, de manera que se determine si esa influencia en el espíritu emprendedor proviene más de un progenitor o de otro, dado que en la investigación de Carlos et al. (2015) se asevera que las acciones de las madres de familia tienen una mayor repercusión en el desarrollo del espíritu emprendedor de sus hijos.

El presente estudio pone de manifiesto que son los progenitores empleados por cuenta propia y ajena los que más dispuestos estarían a proporcionar apoyo económico a sus hijos para montar su propia empresa. Datos concordantes, en parte, con las investigaciones que evidencian que la tradición y el entorno familiar influyen de forma notoria en generador de continuidad o de nuevas actividades empresariales (Arregle et al., 2007 y Bubolz, 2001); e incluso investigaciones realizadas a empresarios en activo (Santamaría et al., 2013) acreditan que el apoyo familiar es un respaldo esencial que ofrece mayores posibilidades de éxito en el negocio.

De modo que, tal y como justifican De la Torre et al. (2016) nos encontramos ante un binomio educación-emprendimiento que puede suscitar prosperidad en el emprendedor, así como en la sociedad y en la economía. En la configuración de este binomio se reconoce el impacto que la educación puede tener sobre el emprendimiento, si bien el conocimiento científico no evidencia si el proceso educativo estimula o no la intención de emprender; sin embargo, sí se ha comprobado que la educación en el emprendimiento es una herramienta indispensable para desarrollar competencias y capacidades emprendedoras (Carlos et al., 2015), bien provenga del ámbito familiar o del escolar y, además, es preciso que sea llevada a cabo en todos los niveles educativos, lo que a futuro, podrá contribuir a crear riqueza y empleo para el bienestar social.

En futuras investigaciones resultaría de interés realizar un análisis longitudinal que compruebe si el fortalecimiento del espíritu emprendedor en el ámbito familiar ejerce una influencia en la creación de nuevas empresas por parte de los hijos. Asimismo, la formación en emprendimiento es uno de los retos fundamentales en la sociedad actual para crear, educar, construir y producir emprendedores (Alemany et al., 2011).

\section{Apoyos}

El texto que presentamos se vincula al proyecto de investigación «De los tiempos educativos a los tiempos sociales: la construcción cotidiana de la condición juvenil en una sociedad de redes problemáticas específicas y alternativas pedagó- 
gico-sociales» (proyecto coordinado EDU2012-39080-C07-00), cofinanciado en el marco del Plan Nacional I+D+i con cargo a una ayuda del Ministerio de Economía y Competitividad, y por el Fondo Europeo de Desarrollo Regional (FEDER, 20072013). También la investigación ha contado con la ayuda puente a Proyectos de Investigación de La Universidad de La Rioja (Ref: APPI 16/09).

\section{REFERENCIAS BIBLIOGRÁFICAS}

Alemany, L., Alvarez, C., Planellas, M. y Urbano, D. (2011). Libro blanco de la iniciativa emprendedora en España. Barcelona: ESADE, Entrepreneurship Institute.

Álvarez Rodríguez, J. y Rodríguez Sabiote, C. (2008). El valor de la institución familiar en los jóvenes universitarios de la Universidad de Granada. Bordón, 60(1), 7-21.

Arregle, J. L., Hitt, M. A., Sirmon, D. G. y Very, P. (2007). The Development of Organizational Social Capital: Attributes of Family Firms. Journal of Management Studies, 44, 73-95. http://doi.org/10.1111/j.1467-6486.2007.00665.x.

Bubolz, M. M. (2001). Family as source, user, and builder of social capital. The Journal of Socio-Economics, 30(2), 129-131.

Carlos Ornelas, C. E., Contreras González, L. E., Silva Olvera, M. Á., Liquidano Rodríguez, M. C. (2015). El Espíritu Emprendedor y un factor que influencia su desarrollo temprano. Conciencia Tecnológica, 46-51. Recuperado de http://4www. redalyc.org/articulo.oa?id=94438997006.

Cuadrado, J. R. y García, A. Emprendedores y Creatividad Competitiva en España. Cuadernos de Ciencias Económicas y Empresariales, 50-51, 13-40.

Davidsson, P. y Honig, B. (2003). The Role of Social and Human Capital Among Nascent Entrepreneurs. Journal of Business Venturing, 18(3), 301-331. http://doi. org/10.1016/S0883-9026(02)00097-6.

De la Torre, T., Luis, I., Escolar, C., Palmero C. y Jiménez, A. (2016). La figura del profesor como agente de cambio en la configuración de la competencia emprendedora. Revista interuniversitaria de formación del profesorado, 86, 131-144. Recuperado de http://revistas.unisucre.edu.co/index.php/rpg/article/view/86/97.

Espíritu Olmos, R. (2011). Análisis de la intención emprendedora en estudiantes universitarios a través de los rasgos de personalidad. Multiciencias, 11, 65-75. Recuperado de http://www.redalyc.org/articulo.oa?id=90418851009.

Flores, M. P. y Palao, J. (2013). Evaluación del impacto de la educación superior en la iniciativa emprendedora. Historia y Comunicación Social, 18, 377-386, http:// doi.org/10.5209/rev_HICS.2013.v18.44256.

Jiménez, A., Palmero, C. y Jiménez, A. (2012). El impacto de educación secundaria y superior en la creación de empresas en la Unión Europea. Revista española de Pedagogía, 252, 201-219.

Ley 15/2011, de 15 de diciembre, de Emprendedores, Autónomos y Pymes. Diario Oficial de Castilla-La Mancha, n 250 de 26 de diciembre de 2011.

Ley $2 / 2012$, de 4 de abril, de apoyo a los emprendedores y las emprendedoras y a la micro, pequeña y mediana empresa. Boletín Oficial de las Islas Balears, no 53 de 14 de abril de 2012. 
Ley 2/2012, de 14 de junio, de Medidas Urgentes de Apoyo a la Iniciativa Empresarial y los Emprendedores, Microempresas y Pequeñas y Medianas Empresas de la Comunitat Valenciana. Diario Oficial de la Generalitat Valenciana, nº 6800, de 20 de junio de 2012.

Ley 16/2012, de 28 de junio, de Apoyo a las Personas Emprendedoras y a la Pequeña Empresa del País Vasco. Boletín Oficial del País Vasco, n 132 de 06 de Julio de 2012.

Ley Foral 12/2013, de 12 de marzo, de apoyo a los emprendedores y al trabajo autónomo en Navarra. Boletín Oficial de Navarra, n 53, de 18 de marzo de 2013.

Ley 5/2013, de 8 de julio, de apoyo a los emprendedores y a la competitividad e internacionalización de las pequeñas y medianas empresas (PYMES) de la Región de Murcia. Boletín Oficial de la Región de Murcia, n 158, 10 de julio de 2013.

Ley 14/2013, de 27 de septiembre, de apoyo a los emprendedores y su internacionalización, Boletín Oficial del Estado, n 233, de 28 de septiembre de 2013.

Ley Orgánica 8/2013, de 9 de diciembre, para la mejora de la calidad educativa (LOMCE), Boletín Oficial del Estado, n² 295, de 10 de diciembre de 2013.

Mwasalwiba, E. S. (2010). Entrepreneurship education: a review of its objectives, teaching methods, and impact indicators. Education + Training, 52(1), 20-47. http://doi.org/10.1108/00400911011017663.

Orrego Correa, C. I. (2009). Fenomenología y emprendimiento. Revista Pensamiento \& Gestión, 27, 235-252.

Osorio, F. F. y Pereira, F. (2011). Hacia un modelo de educación para el emprendimiento. Una mirada desde la Teoría Social Cognitiva. Cuaderno de Administración, 24(43), 13-33.

Santamaría, A. E., Moreno, E. M., Torres, E. F. y Cadrazco, W. (2013). La influencia de la familia en el emprendimiento empresarial - El caso de tres pymes de los sectores comercial y de servicios de le ciudad de Sincelejo Sucre. Revista Pensamiento Gerencial, 1.

Seikkul-Leino, J., Ruskovaara, E., Ikavalko, M., Mattila, J. y Rytkola, T. (2010). Promoting entrepreneurship education: the role of the teacher? Education + Training, 52, 117-127. http://doi.org/10.1108/00400911011027716.

Taktak, S. y Triki, M. (2015). The Importance of Behavioral Factors: How Do Overconfidence Affect Entrepreneurial Opportunity Evaluation? Journal of Behavioural Economics, Finance, Entrepreneurship, Accounting and Transport, 3(1), 12-20. http://doi.org/10.12691/jbe-3-1-2.

Vican, D. y Luketić, D. (2007). Self-assessment of croatian elementary school pupils on the entrepreneurial initiative. Journal of Contemporary Management Issues, 18(2), 57-79.

Von Graevenitz, G., Harhoff, D. y Weber, R. (2010). The effects of entrepreneurship education. Journal of Economic Behavior and Organization, 76(1), 90-112. http:// doi.org/10.1016/j.jebo.2010.02.015. 